Gamma-Free Neutron Detector Based upon Lithium Phosphate Nanoparticles-

Neutron Sciences, Inc., 1256 Lovell View Drive, Knoxville, TN 37932-2592; 865-7770273, www.neutronsciences.com

Mr. Steven A. Wallace, Principal Investigator, swallace@neutronsciences.com

Ms. Marie Allison, Business Official, info@neutronsciences.com

DOE Grant No. DE-FG02-06ER84637

Amount: $\$ 99,890$

A gamma-free neutron-sensitive scintillator is needed to enhance radiation sensing and detection for nonproliferation applications. Such a scintillator would allow very large detectors to be placed at the perimeter of spent-fuel storage facilities at commercial power plants, so that any movement of spontaneously emitted neutrons from spent nuclear fuel or weapons grade plutonium will be noted in real-time. The material must have an efficiency greater than that presently available using scintillating glass fibers and helium-3 tubes, but the most important need is for a detector material that is totally free of gamma interference. This project will develop technology for manufacturing large panels of fluor-doped plastic containing lithium-6 phosphate nanoparticles. In order to detect neutrons, the nanoparticles must be sufficiently small so that the plastic remains transparent. In this way, the triton and alpha particles generated by the capture of the neutron will result in a photon burst that can be coupled to a wavelength shifting (WLS) fiber, producing an optical signal of about ten nanoseconds at the face of a multianode photomultiplier. In Phase I, a patent-pending process for manufacturing the lithium-6 phosphate nanoparticles will be characterized, and the feasibility of embedding the nanoparticles in a plastic scintillator material will be determined.

Commercial Applications and other Benefits as described by the awardee: The lithiated plastic should find application to the Linac-based x-ray systems being used at ports of entry for the imaging of cargo located in sea-land containers. The plastic detectors would enable a real-time determination as to whether a container has any fissile material hidden within high density material. 


\section{Project Narrative}

\section{(1) Identification and Significance of the Problem or Opportunity}

Neutron detection is often made using helium-3 tubes and using cerium activated lithium glass fibers. Both of these technologies have difficulty in scaling to larger size detectors. Straw detectors are being pursued, but to obtain large area detectors using this technique is also very difficult.

A promising concept of using plastic scintillators to allow neutron detection has been developed at the Fermi National Accelerator Laboratory. The basis for the detector is the method used for fabricating a large neutrino detector. The detector is named MINOS and is composed of 330 tons of polystyrene containing scintillation fluors. The detectors are made from a massive number of extruded polymeric bars having a one centimeter by four centimeter cross section and an extrusion length of eight meters. Sixty four of the bars are coupled using wavelength shifting fibers to an $8 \times 8$ pixel Hamamatsu photomultiplier. Using the MINOS detector as a model for developing a large neutron detector allows realistic projections to be made as to the possibility of mimicking the technical approach to the detection of neutrons. The primary limiting technology for developing such large area detectors is the availability of highly efficient and economically feasible polymeric scintillators. As will be noted later in this proposal, a just completed DOE Phase I SBIR grant has successfully demonstrated using lithium-6 phosphate nanoparticles in clear plastic in cast and thin film forms. The measured results show a significant potential for developing future solid-state neutron detectors that are highly efficient, have near zero false positive alarms, and are economically manufacturable.

The crucial results demonstrated in the DOE Phase I is that clear plastic films and castings have been demonstrated that have a high optical transmission even containing dopant particles which are highly efficient at capturing neutrons. The approach that is being pursued in this grant application is the dispersion of nanoparticles of lithium-6 phosphate within a clear plastic coupled to a prototype neutron detecting system. The DOE SBIR used PMMA as the base polymeric material. With a 940 barn neutron capture cross section and the production of a triton and an alpha particle having a combined kinetic energy for deposition of $4.8 \mathrm{MeV}$, a very useful neutron detecting material was generated. The very small size of the nanoparticles, less than 50 nanometers in diameter, allowed a loading of the particles at about a three percent weight with retention of transparency.

Demonstrating that the lithium-6 nanoparticles can be dispersed into a clear plastic containing fluors and that scintillation does occur was the technical challenge that was demonstrated in the DOE Phase I SBIR. This SBIR builds upon this foundation by demonstrating the solid-state neutron detecting material can be used for the manufacture of a portable neutron detection system. One system will use a five inch photomultiplier to demonstrate a hand portable neutron detector and the second system will use a back pack portable system using the neutron detecting material coupled to sixty four slabs of 
the plastic having wavelength shifting fibers coupling the scintillation pulses to an $8 \mathrm{x} 8$ pixel Hamamatsu photomultiplier. This latter technology would be for the building of detectors for placement at the side walls of vans and would allow the detection of a spontaneous weapons grade plutonium neutron emitter of five kilograms weight at one hundred yards in one minute.

\section{(2) Phase I Technical Objectives}

This Phase I grant proposal is designed to unambiguously demonstrate that the use of lithium- 6 phosphate nanoparticles in a PMMA or similar polymer matrix can allow the development of two neutron detecting systems resulting in improved security of the United States of America.

The technical objectives of this Phase I Project is to fully characterize the neutron detecting materials as a system for detecting a neutron source located many meters away, and to demonstrate that the use of films and thin castings provide a cost effective manufacturing technology. The goal is to demonstrate that a commercial product can be cost effectively built and fielded for the monitoring of neutrons sources located at spent fuel transfers, at loading docks, and other related areas for sensitive detection of neutrons.

\section{(3) Phase I Work Plan}

Neutron Sciences will immediately upon receipt of grant funding embark upon the manufacture of approximately one kilogram of lithium- 6 phosphate nanoparticles. Demonstrating that such a quantity of material can be manufactured rapidly will assure that commercialization of the process of making the basic material for the neutron detectors are feasible.

Neutron Sciences will develop the thin films on a suitable substrate for efficient optical coupling to a five inch photomultiplier tube. The electronics will be manufactured that will allow a hand held portable package to be tested. Neutron Sciences will also explore the wavelength shifting fibers (WLS) cast within polymethyl methacrylate containing one percent by weight PPO/POPOP and will have built by Telesenors, a small start-up company, the circuitry needed to measure neutrons from sixty four castings simultaneously using WLS to couple the scintillation pulses to a small $8 \times 8$ pixel PMT. This demonstration is important to show that large area detection can be built up as modules so that several square meters of detection area is possible.

Testing of the neutron detectors will be done by Neutron Sciences at the University of Tennessee as the plutonium-beryllium source will be raised up and out of the shielding drum so that neutrons will be a point source similar to what a spent fuel rod will be. The bar detectors will be located up to thirty feet away. In addition, a small Californium-252 source may be used at the radiation waste storage facility at the University of Tennessee Hospital if arrangements can be made. This facility is remote and backs up on a high bank of the Tennessee River. Dr. Laurance Miller from 
University of Tennessee will help Neutron Sciences in providing neutron point source option for large area detector testing.

A very portable system can be demonstrated for the detection of neutrons by basically covering a five inch photomultiplier tube with the material already demonstrated in the DOE Phase I. An entire system with readout can be built within a box eight inches square by just over twenty inches long. This is about the size of the portable germanium gamma detectors being delivered to the Department of Homeland Security. Essentially an instrument complementary to the gamma detector for the detection of neutrons can be fielded by just extending the work achieved in the DOE Phase I.

Upon the completion of the work outlined above, Neutron Sciences will be in a position for setting up a manufacturing process for the building of large, highly efficient, gamma-free neutron detectors. The photomultiplier based solid-state neutron detectors are passive, have low power requirements, and are benign in their need for servicing. See the following link at:

http://www.osti.gov/bridge/product.biblio.jsp?query_id=1\&page=0\&osti_id=835543

for an analysis made in the earlier SBIR of the sensitivity calculation for detecting weapons grade plutonium.

Performance Schedule

1. Conversion of one kilogram of lithium- 6 as the monohydrate to lithium- 6 phosphate nanoparticles in batches of twenty grams for blending is the three plastic types to be tested with the WLS electronics

\section{first two months}

2. Development of five inch diameter plastic scintillator thin films made using the three forms based upon the MMA, PS/PPV and PVT demonstrated in the DOE Phase I SBIR. Determine which form is suitable as a manufacturing technique for optimum scintillation output into the face of a five inch photomultiplier.

second and third months

3. Testing of the five diameter scintillators with PMTs and the concurrent building of the electronics for the processing of the simultaneous measurement of multiple readouts at the University of Tennessee Nuclear Engineering Department.

fourth and fifth months

4. Test a prototype detecting system containing sixty four cast plastic blocks, again using the three different plastic bases of the neutron detecting material coupled to the wavelength shifting fibers coupled to a Hamamatsu sixty four pixel photomultiplier at the University of Tennessee Nuclear Engineering Department

third through sixth months 
7. Testing the five inch PMT hand portable neutron detection system with all electronics located within an $8 \times 8 \times 14$ inch box at the University of Tennessee Nuclear Engineering Department.

$$
\text { third through sixth months }
$$

8. Testing and completion of test program demonstrating that a solid-state neutron detectors composed of three different plastic bases containing lithium phosphate do work in two system configurations and that such systems can be manufactured reliably and cost efficiently. Determine which of the three base plastics functions the best.

$$
\text { sixth month }
$$

\section{(4) Related Work}

A DOE Phase I technical feasibility has just been demonstrated of the plastic neutron detection material. The steps for manufacturing lithium-6 phosphate nanoparticles developed at Oak Ridge National Laboratory were duplicated. Nine grams of lithium- 6 phosphate nanoparticles were made by Neutron Sciences.

Lithium-6 monohydrate was purchased from the Isotope Sales Office of the Oak Ridge National Laboratory. The first step was the chemical transformation of the monohydrate form of the lithium to the oleate. To increase the content of neutron target isotope, the lithium- 6 containing nanoparticles were prepared using enriched lithium-6 and used instead of the naturally abundant lithium isotopes (7.42\% Li-6, 92.58\% Li-7).

Samples of $\mathrm{Li}_{3} \mathrm{PO}_{4}$ nanoparticles with particles sizes around $30 \mathrm{~nm}$, were prepared (Fig. 1) by the direct reaction between lithium salts dispersed in a long chain carboxylic acid and dry phosphoric acid. The photograph taken at Oak Ridge National Laboratory was augmented by the ORNL staff with the preparation of five samples of the nanoparticles being embedded in PVT using the process that ORNL developed for the Department of Homeland Security. The process used by Neutron Sciences used standard laboratory reactions which proceeded using hotplate temperatures to yield nine grams of crystalline nanoparticles. Four grams of the nanoparticles were delivered to Dr. Adam Rondinone at ORNL for characterization and the preparation of disks for placement on the end of the PMT and five grams were retained by Neutron Sciences. The Neutron Sciences nanoparticles were made into thin films on three quarter inch quartz disks. These thin films were then tested in the NUMEC neutron Howitzer to evidence the generation of scintillation pulses due to neutron capture in the lithium-6. About forty different quartz disk samples were prepared at the University of Tennessee for testing using different fluors and different loadings of the nanoparticle density. All of the results showed that the thin film disks generated detectable scintillation pulses.

The method of testing the disks was to lower the disk having the film of neutron detecting material coupled to the PMT into the irradiation port. The spectrum of the 
scintillation pulses was taken for twenty seconds and the gross counts in the spectrum were counted. The measurement was made every inch from the bottom of the port to the outside of the neutron Howitzer in one inch steps. In this way relative comparisons were made of the efficiency of the detection material between the fluors and the loading densities could be made. The results were positive and did show that higher loading of the nanoparticles did result in higher counts. The comparison between the fluors needs further investigation as the results contained unanswered questions as to to what the gram loadings were. Such ambiguities are a part of experimental work done in a short time.

The first step was the production of the lithium- 6 oleate. This proceeded by the following steps. Six grams of $6 \mathrm{LiOH} \cdot \mathrm{H}_{2} \mathrm{O}(146 \mathrm{mmol})$ was dispersed in $200 \mathrm{~mL}$ ethyl alcohol (200 Proof), 45.80g oleic acid $(146 \mathrm{mmol})$ was added and refluxed overnight. Finally the lithium oleate was recrystallized in boiling ethyl alcohol.

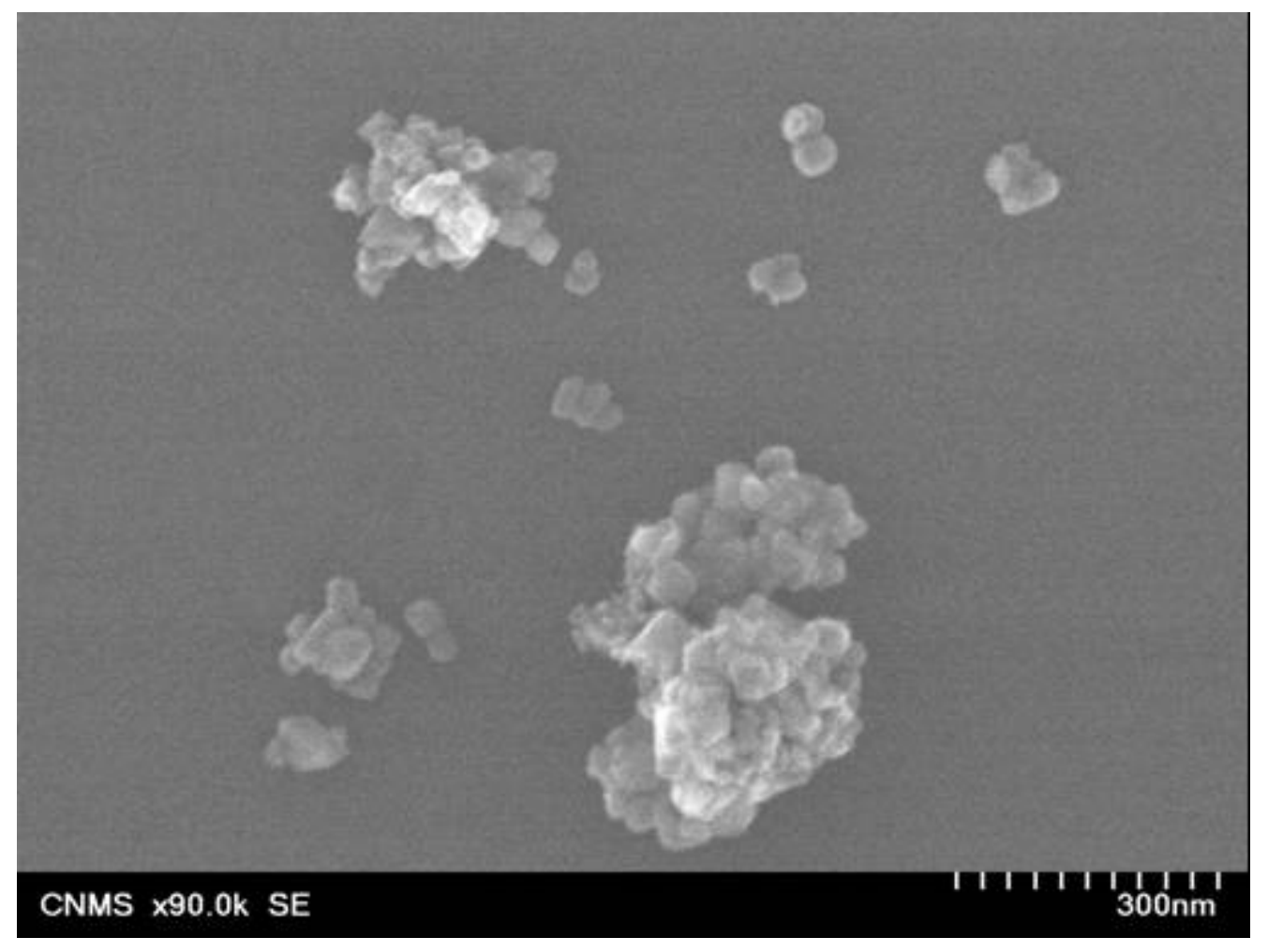

Fig. 1: $\mathrm{Li}_{3} \mathrm{PO}_{4}$ Nanoparticles (Particles Sizes $\approx 30 \mathrm{~nm}$ )

The synthesis of $\mathrm{Li}_{3} \mathrm{PO}_{4}$ from lithium oleate $(1.5 \mathrm{~g})$ proceeded by dissolution in a mixture of phenyl ether $(10 \mathrm{~g})$, tributyl phosphate $(5 \mathrm{~g})$, oleic acid $(3 \mathrm{~g})$, and trioctylamine $(4.5 \mathrm{~g})$ at $140^{\circ} \mathrm{C}$. A solution of phosphoric acid $\left(0.5 \mathrm{~g}, 35 \% \mathrm{H}_{3} \mathrm{PO}_{4}\right.$ solution) in hexylether was then added. The heat was removed immediately and the reaction mixture was stirred for $12 \mathrm{~h}$. The surface modification of $\mathrm{Li}_{3} \mathrm{PO}_{4}$ nanocrystals was done in-situ. The in-situ surface modification of the nanocrystals resulted from the reaction mixture being heated to $100^{\circ} \mathrm{C}$. and a mixture of dodecylamine $(2.8 \mathrm{~g})$, hexyl ether $(4.0 \mathrm{~mL})$, and bis (2-ethylhexyl) hydrogen phosphate $(5.0 \mathrm{~mL})$ being added. The surface modification was allowed to proceed at $100^{\circ} \mathrm{C}$. for $1 \mathrm{~h}$. Then, the working up of 
the reaction was done by adding anhydrous isopropyl alcohol to precipitate $\mathrm{Li}_{3} \mathrm{PO}_{4}$ nanocrystals. The nanocrystals were washed three times with fresh, anhydrous isopropyl alcohol. Surface modified $\mathrm{Li}_{3} \mathrm{PO}_{4}$ nanoparticles were found to be highly dispersible in organic solvents, e.g., toluene.

United States patent application 2006054863, March 16, 2006, Composite scintillators for detection of ionizing radiation contains the above noted method of making the lithium- 6 phosphate nanoparticles. This patent is filed by UT-Battelle as the operator of the Oak Ridge National Laboratory and Neutron Sciences is a licensee to the technology.

Transparent to translucent samples of neutron scintillators were made from the combination of Li-containing nanoparticles as neutron target material and a scintillator embedded in suitable polymeric matrix. Different organic scintillator combinations were tested as films on quartz disks. This development of very sensitive neutron detectors uses unique procedures custom developed in this study for optimal dispersion of neutron sensitive nanoparticles in a matrix of a polymer or a blend of polymers/co-polymers to obtain thin film scintillators, fiber based scintillators, and bulk-composites neutron scintillation counters and screens without affecting the optical transmission properties of the composites and avoiding agglomeration of scintillation nanoparticles. Significant work on this end was developed by Professor Dayakar Penumadu from University of Tennessee, Knoxville, and his doctoral student Mr. Indraneel Sen working closely with Mr. Steven Wallace of Neutron Sciences Inc.

Monolithic sample of lithium-containing nanoparticle, an organic scintillator PPO, and a wavelength shifter POPOP, embedded in polyvinyl toluene gave large scintillation pulses upon the exposure to thermal neutrons using a plutonium-beryllium (Pu-Be) neutron source available at the University of Tennessee, Knoxville. The scintillators embedded with lithium-containing nanoparticles were non-hygroscopic despite the hygroscopic nature of lithium-containing compounds.

The DOE Phase I effort related to finding an optimal dispersion of lithium nanoparticles in the form of ionic salts in a blend of polymer/copolymer matrix. Lithium intercalated fullerene molecules can also be used instead of a lithium salt. The optimal dispersion of the nanoparticles increases the efficiency of the scintillation counter several folds over the cerium glass currently available as the choice neutron scintillation material. The data taken during the DOE Phase I show that economical, efficient and flexible thin film neutron scintillation devices as thin as a few tens of microns can be synthesized using this technique.

The first of three methods of preparing test samples entailed mixing a commercial blend of organic phosphors PPO/POPOP dissolved in methyl methylacrylate (MMA) solvent that has been distilled to extract the inhibitors. Lithium phosphate nanoparticles are then suspended in the solution by ultrasonication. Initiators are added in the resulting suspension and the MMA is partially polymerized in the ultrasonic bath till the media becomes viscous and the suspension is stabilized. Partially polymerized suspension is 
removed from ultrasonic bath and allowed to completely polymerize either by heat or by ultra violet curing. The polymer cylinder thus obtained by breaking the reaction vial is then ready to be processed. It can be palletized to be extruded into appropriate shapes (e.g. rods, fibers, films etc.) or directly processed by a lathe or shaping machine to create various shapes (e.g. disks, cubes, prisms etc.).

The second method mixes in a $25 \mathrm{ml}$ vial the lithium salt nanoparticles, high molecular weight polystyrene (PS) and a blend of polyphenylene vinylene (PPV) based light emitting copolymers dissolved in chloroform by ultrasonication. A few drops of the solution are used to spin coat a thin film on a quartz or silicon wafer substrate.

A third method of was a preparation of $3.1 \mathrm{wt} \% \mathrm{Li}_{3} \mathrm{PO}_{4}$ nanoparticles in polyvinyl toluene (PVT) monoliths, $15 \mathrm{mg}$ of nanoparticles were dispersed in $450 \mathrm{mg}$ of methylstyrene (99\%, Aldrich, freshly distilled over $\mathrm{CaH}_{2}$, kept under $\mathrm{N}_{2}$ ). A free-radical initiator (2, 2'-Azodi (2,4'-dimethylvaleronitrile)) and PPO/POPOP mixture both at $1 \mathrm{wt}$ $\%$ of methyl styrene was then added to the $\mathrm{Li}_{3} \mathrm{PO}_{4} /$ methylstyrene dispersion. The transparent dispersion was purged with $\mathrm{N}_{2}$ gas and the free-radical initiated polymerization was allowed to proceed at $36^{\circ} \mathrm{C}$ for a week.

The photomultiplier tube (PMT) used was a 3/4" head-on Hamamatsu R1464 biased at a negative 1000 volts. This tube was selected to allow the transport of the tube down the port channel of the NUMEC neutron Howitzer located at the Nuclear Engineering building at the University of Tennessee. The PMT is powered by a Hamamatsu Socket Assembly E974-13. The signal is an input to an Ortec Shaping Amplifier set to a 0.1 microsecond time constant, a negative input, a $20 \mathrm{X}$ coarse gain, a 0.5 fine gain and a unipolar output. The output goes to an Ortec PCI based multi channel analyzer (MCA) from which the spectrum of pulse heights are stored, recorded and analyzed.

Samples are mounted on the PMT using Bicron optical grade. The sample is then covered by a plastic cylinder made light tight using black PVC tape to stop prevent light leakage. The background signal is then checked and recorded by running the PMT. The PMT is lowered in one inch steps into a 16 " cavity in a drum containing the plutoniumberyllium neutron source. At each step location, the detector response to the neutron field is measured for twenty seconds by recording all of the scintillation pulses using an Ortec PCI multichannel analyzer. After the neutron measurements are completed, the PMT with the sample attached and with the sample removed is measured for gamma response using one microCurie Co-60 source and a one microCurie Cs-137 source. Gamma counts are noted at about one percent of the level of the counts seen with the detector sample at the bottom of the irradiation sample, but of more significance is the fact that the gamma response is the same with and without the sample clearly showing that the gamma counts are due to an interaction in the tube and not in the sample. 

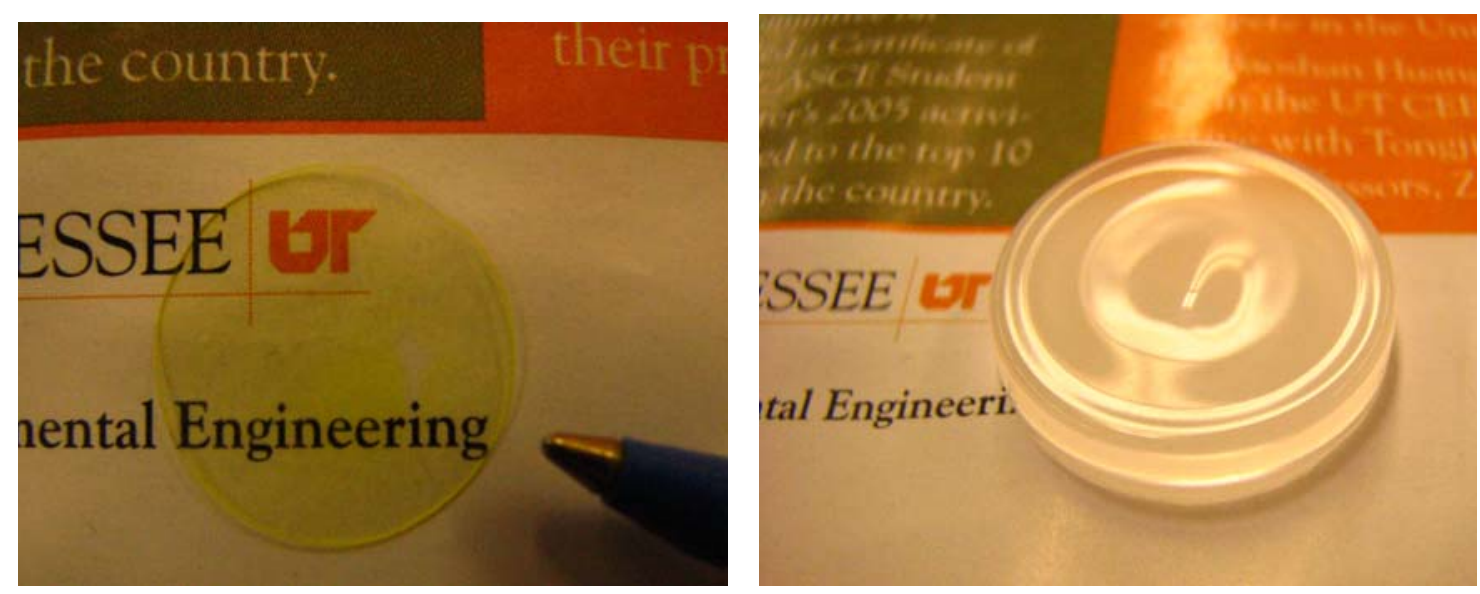

Figure 2 (a) shows a thin film scintillation detector (on left) made by spin coating a blend of polystyrene and a polyphenylene vinylene copolymer (with peak photoluminescence at $500 \mathrm{~nm}$ ) with suspended lithium phosphate nanoparticles in chloroform solution placed upon a 3/4" transparent quartz disk. Figure 2 (b) shows a solid $3 \mathrm{~mm}$ disc (on right) of PMMA with dispersed lithium phosphate nanoparticles and containing a blend of organic polymer BMPPV and the commercial oligomer ADS086BE.

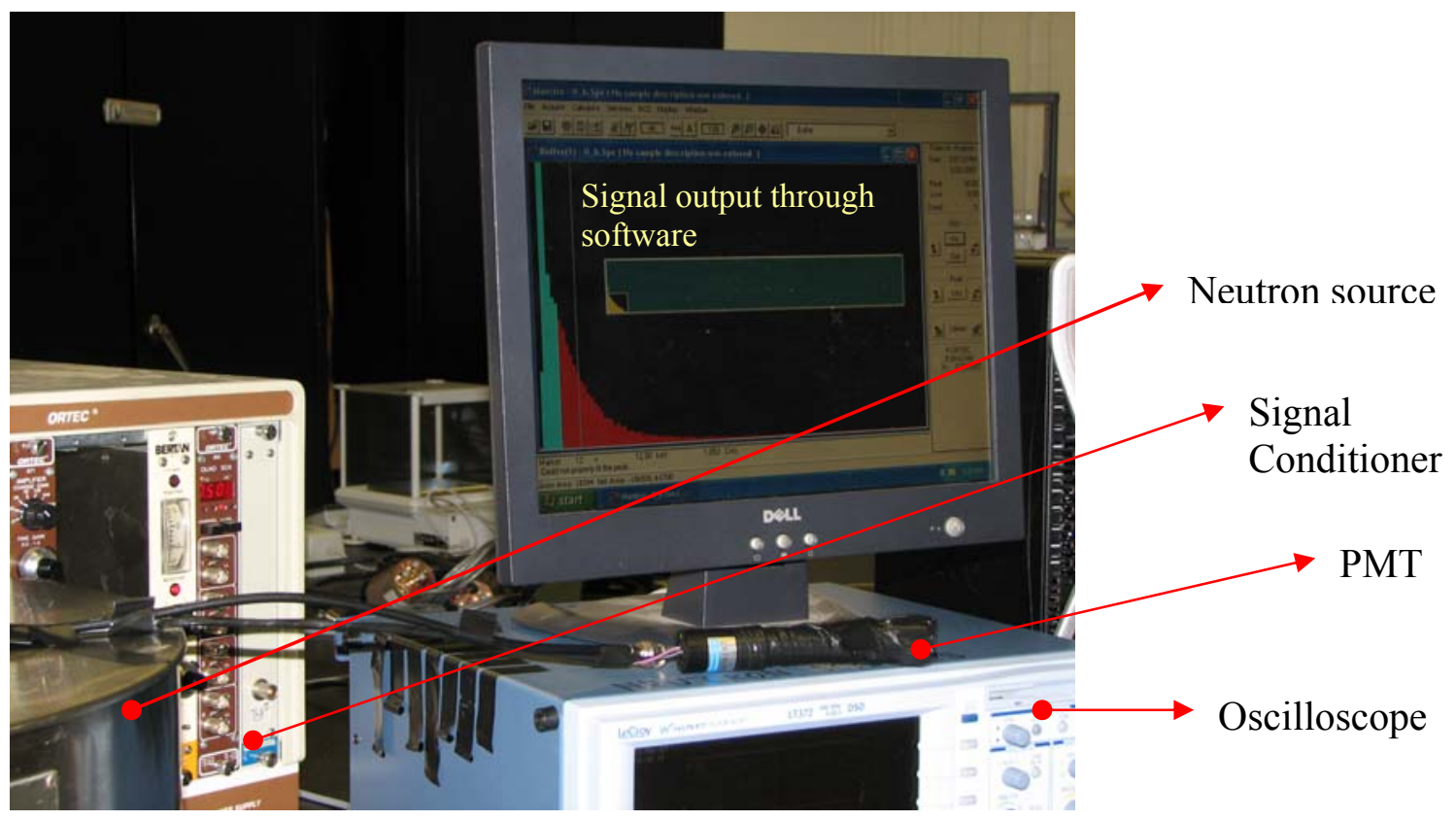

Figure 3. Experimental setup showing edge of neutron containing source drum, the Ortec 671 amplifier and high voltage in a miniNIM bin, the photo multiplier onto which the $3 / 4$ " diameter thin films and the cast disks are placed and the LeCroy digital oscilloscope. Not shown is the computer containing the Ortec PCI based multichannel analyzer. 


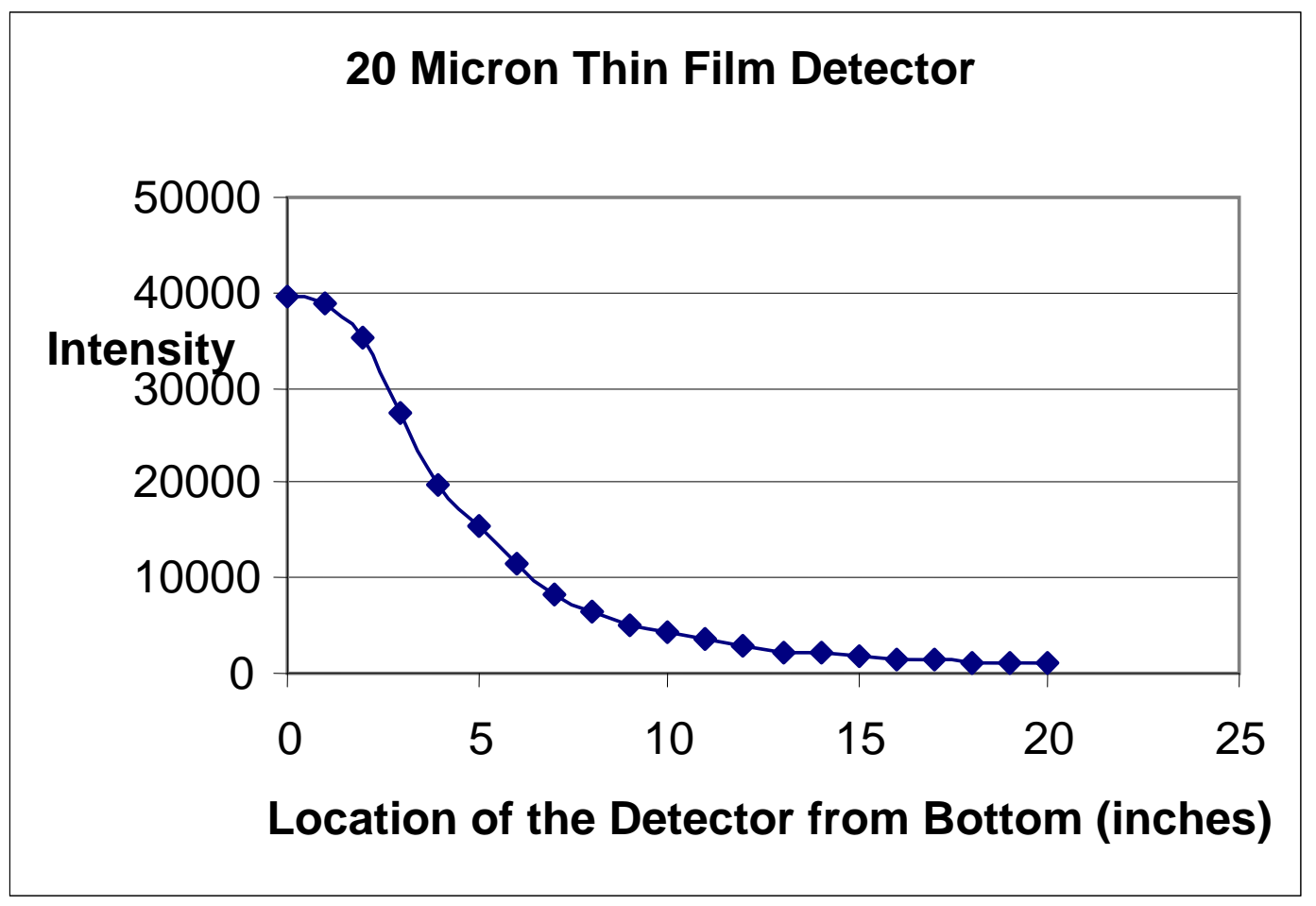

Figure 4 Stable scintillation characteristics of $20 \mu \mathrm{m}$ thick plastic scintillator

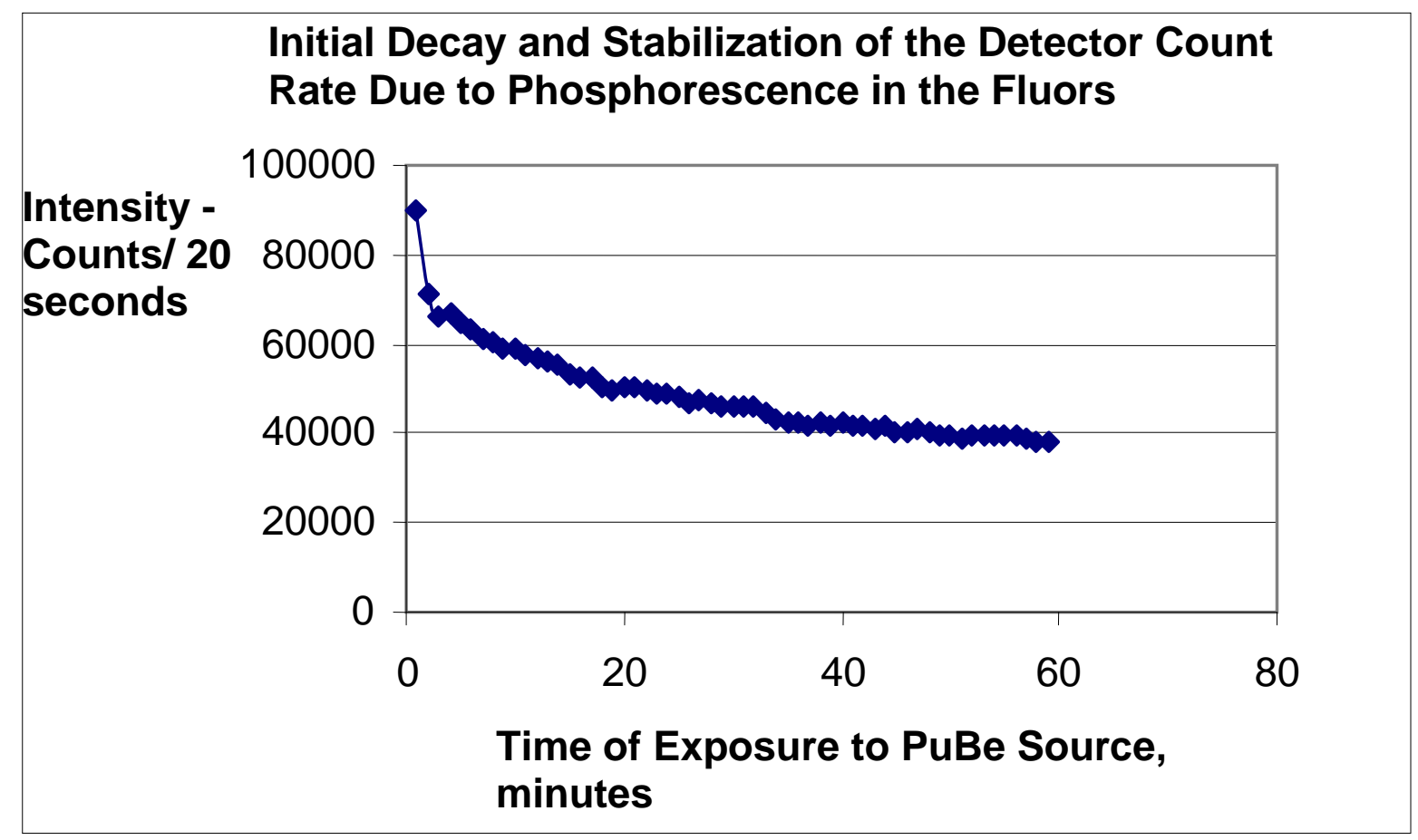

Figure 5 Initial decay and subsequent stabilization of thin film detector 


\section{Scintillator Performance after 1 Day of Exposure to PuBe Source}

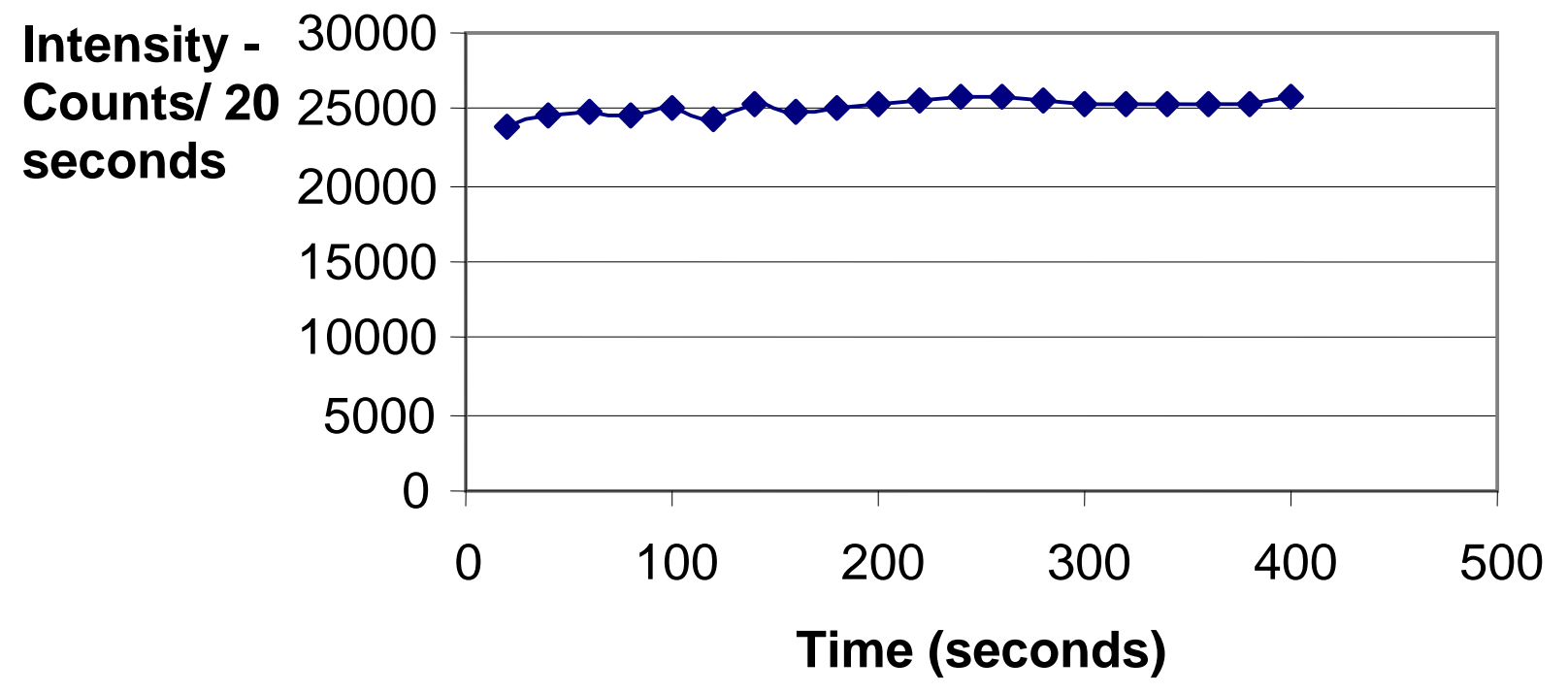

Figure 6 Stable response of the thin film detector after 1 day of $\mathrm{Pu}$-Be source exposure

Figure 4 is typical of the data that results from the measurement of the neutron counting efficiency of the thin films tested in the DOE Phase I program. A thin film of PMA containing about three weight percent of the lithium phosphate nanoparticles and about one weight percent of the fluor yielded about 40000 neutrons detected in a twenty second interval or detected neutrons at a yield of $2000 \mathrm{n} / \mathrm{s}$. Multiple samples were tested of films and cast disks with results showing a low detection rate of about $1500 \mathrm{n} / \mathrm{s}$ to about $2500 \mathrm{n} / \mathrm{s}$. This should be compared with data taken using a surface barrier detector (SBD) coated with lithiated glass reported in Applied Radiation and Isotopes 53 (2000) 755-758. The SBD was lowered into the same well in the NUMEC neutron irradiator but had only $350 \mathrm{n} / \mathrm{s}$ detection efficiency. The SBD was a 450 square $\mathrm{mm}$ detector compared with the 300 square $\mathrm{mm}$ of the disks used on the photomultiplier tube giving an efficiency of the SBD of about $80 \mathrm{n} / \mathrm{s} / \mathrm{cm}^{* *} 2$ compared with an efficiency of at least 500 $\mathrm{n} / \mathrm{s} / \mathrm{cm}^{* * 2}$.

Figure 5 shows an unexpected result that reveals a significant difference between the samples prepared as shown in Figure 2 (a) and Figure 2 (b). Phosphorescence occurs in the samples prepared in the polyphenylene vinylene copolymer. The room light stores energy in the samples which bleeds out over several hours. Whereas the clear plastic samples cast into pellets as seen in one sample shown in Figure 2 (b) does not exhibit any residual light and the scintillation response that is seen immediately upon using the detector material stays the same. 
Figure 6 shows that the output of the thin coating of neutron absorbing film on quartz does reach a steady-state responsitivity to the detection of neutrons after 24 hours.

Figure 7 shows the response of a $3 \mathrm{~mm}$ diameter and $3 \mathrm{~mm}$ thick lithiated glass detector using the same source described above as a function of its location from the bottom of the drum corresponding to source location. GS-20 glass is one of the best neutron detectors that are commercially available and use in the current practice and are very expensive.

It is very interesting to note that our new nanoparticle based thin film detector produced approximately fifty percent of the counts of a $3 \mathrm{~mm}$ diameter $\times 3 \mathrm{~mm}$ high GS20 glass cylinder. In terms of counts per unit volume, our thin film uses very little material to generate $50 \%$ of the responsivity of a very expensive glass detector. The film is only an area of a 3/4" spot and can easily be developed to cover completely the face of a five inch photomultiplier tube. The counts from this area of thin film detector would be very low cost compared to a very high cost of making the glass for large area, further demonstrating the great potential of the present technology developed during the DOE Phase I SBIR grant.

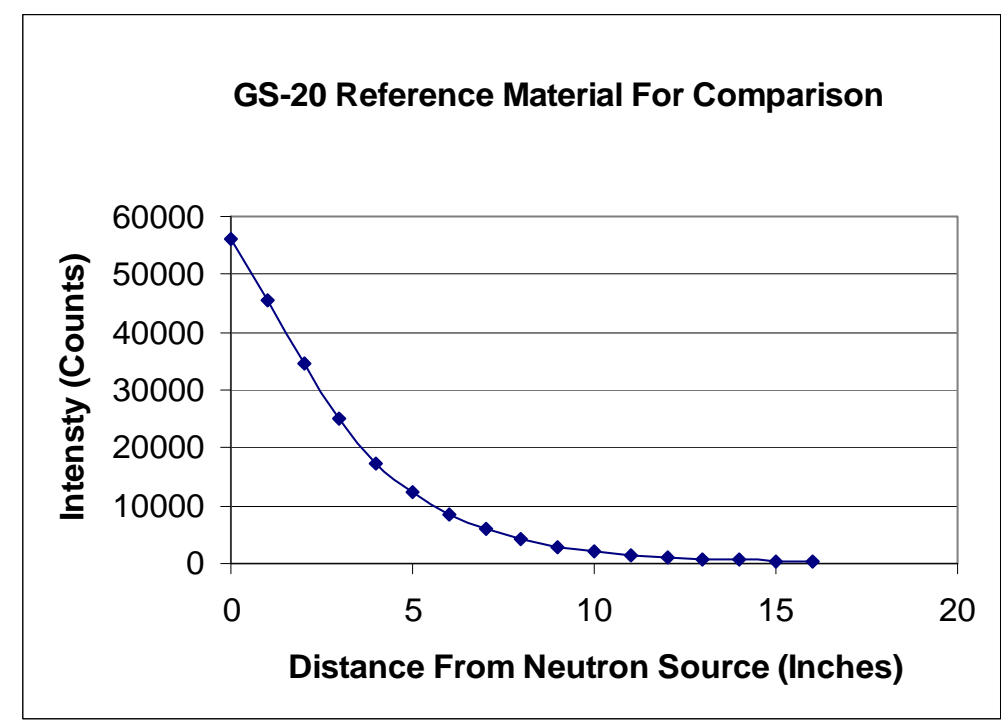

Fig.7: Scintillation Response of 3mm x 3 mm GS 20 (Lithiated Glass for Comparison)

Andrew Stephan in his Ph.D. Thesis from Nuclear Engineering program at the University of Tennessee, Knoxville (http://etd.utk.edu/2003/StephanAndrew.pdf), examined the efficiency of distributing nanoparticles within thin films. His calculations using the TRIM code (www.srim.org) developed by James Ziegler to determine the efficiency of the energy transfer of particles from the stopping of the alpha particle and the triton show that one hundred percent efficiency occurs for lithium phosphate particles less than one hundred nanometers. There is essentially no loss of energy within the particles themselves so that the entire energy is deposited within the plastic. Furthermore the capture cross section of the lithium- 6 is such that essentially all thermal neutrons will be absorbed within a few tens of microns of solid lithium. The results that have been 
obtained with the loading of the lithium- 6 phosphate nanoparticles show that very efficient detection of neutrons has been demonstrated for thin films and castings of clear plastic less than five $\mathrm{mm}$ in thickness.

Neutron Sciences met the objective of Phase DOE grant demonstrating that efficient, gamma-free, detection of neutrons is possible using lithium-6 phosphate as the neutron absorber. By placing nanoparticles of this neutron absorbing material within clear plastic doped with scintillation fluors highly efficient neutron detection occurs, and detectors can be produced with large area and high sensitivity and can be obtained in geometric shapes as needed for its broad implementation as next generation neutron detectors.

\section{(5) Relationship with Future Research or Research and Development}

Successful demonstration of a solid-state neutron detector system based upon a plastic containing lithium phosphate nanoparticles and a scintillating fluor will provide the groundwork for the manufacturing a useful neutron detector capable of being used by the agencies of the government needing such instruments. By demonstrating the first prototypes capability the foundation is made for seeking the Phase II funding for the development of an actual production model of the neutron detector.

\section{(6) Commercialization Strategy}

Neutron Sciences has been developing the technology for the manufacture of solid-state neutrons detectors supported by Small Business Innovation Research grants. Up until the completion of the most recent DOE supported grant for the demonstration that lithium phosphate nanoparticles could be dispersed within a clear plastic, no really commercial development had been achieved. The results obtained with the films and castings manufactured in the just completed Phase I DOE SBIR detected neutrons with an estimated efficiency of twenty percent and did not respond to the gamma rays from Cobalt-60, Cs-137 or Ba-133. In principle castings of the material could be marketed now. Three different plastics were demonstrated in the Phase I DOE SBIR. This grant will allow the plastics to be evaluated using wavelength shifting fibers and electronics which can be commercialized.

Neutron Sciences seeks to work with Telesensors to make a completed instrument for detecting neutrons. Two products are to be manufactured and offered for sale.

This grant is based upon achieving two objectives. The first is to determine which of three plastics functions the best as a neutron detector. The second objective really is needed for determining the first objective and is to build two prototype detectors that will allow the determination of the best plastic to be determined based upon real hardware. The first prototype is a handheld detector similar in size to the mechanically cooled high purity germanium gamma detectors manufactured by Ortec. The design will be that of a box approximately eight inches by eight inches in cross section and about sixteen inches long. This is a size that can be easily used for scanning vehicles, crates or boxes. The 
neutron detection will be made by a film or casting of about two mm thickness of the clear plastic, most likely polymethyl methacrylate, containing the scintillation fluors PPO/POPOP at about one weight percent and the lithium phosphate nanoparticles at about three weight percent. This combination works very well as has been demonstrated in the just completed DOE SBIR Phase I grant. Telesensors is a small company having several Ph.D. electrical engineers. Neutron Sciences has a suite next to Telesensors at the Fairview Technology Center in west Knox County, Tennessee. Telesensors will build the circuitry for the processing of pulses from the five inch photomultiplier tube that has the thin film or casting of clear plastic attached to the PMT face. This Phase I request is instrumental in funding the development of this packaging and circuit design so that a prototype can be demonstrated.

The second prototype is to cast the three plastics around wavelength shifting fibers so as to allow a large area detector to be built. Telesensors, Inc. will build the electronics for the detection of any pulses arriving at any one of the sixty four output lines coming from a Hamamatsu $8 \times 8$ pixel PMT. This second prototype is very important because should any one of the three plastics allow detection of neutrons using the wave shifting fibers, then very large detectors can be built.

Once the prototypes are demonstrated, venture capital funding will be sought using the contacts that are available through the Tech 2020 organization (www.tech2020.org). Since the lithium phosphate nanoparticles being manufactured by Neutron Sciences is a licensed product being commercialized from the Oak Ridge National Laboratory, the full resources of the Tech 2020 organization is available. Manufacturing space at the Fairview Technology Center will be sought from the Tech 2020 organization so that Telesensors and Neutron Sciences can hire the personnel to set up a modest production line for the manufacture of the detectors.

Technology 2020 houses a Small Business Development Resource Center where consultants can help small business owners with questions about operations, manufacturing, engineering, personnel, administration, marketing, sales, finances, business plans and other activities. Free counseling services on starting a business, building a business, and related topics are available. Neutron Sciences is a member of Tech 2020 as a licensee of Oak Ridge National Laboratory technology. The full resources of this organization will be used to map out the capital needed to place the detectors into a production mode.

Commercialization will need to be demonstrated at the prototype level to get any venture capital investment. The prototypes to be demonstrated in this Phase I will need to be refined in a Phase II to show that the solid-state plastic detector can be shown to have at least a twenty percent neutron capture efficiency. In addition, the Phase II will demonstrate that detectors of several square meters can be built using long plastic blocks up to eight meters in length. This is the length of the plastic blocks used at the Fermi National Accelerator Laboratory MINOS detector. Such large detectors can be placed in trucks that will have the sensitivity to detector neutron sources hundreds of meters away. 


\section{(7) Key Personnel}

Steven Wallace - Principal Investigator for Neutron Sciences, Inc.

BS 1963 Physics Purdue University

Neutron Sciences was formed to commercialize the sol-gel based manufacture of lithiated glass licensed from the Oak Ridge National Laboratory. This SBIR is based upon a license from ORNL for the manufacture of the nanoparticles of the lithium phosphate and this submission is to demonstrate that large neutron detectors can be fabricated from the material as a scintillation generator upon the absorption of neutrons by the lithium- 6 .

This nanoparticle detector material was developed at Oak Ridge National Laboratory under NA-22 support with Dr. Adam Rondinone as the PI. Dr. Suree Brown and Dr. Sheng Dai of the ORNL also participated in the development. Steven Wallace contributed to the patent application (20060054863) with Dr. Dai and Dr. Rondinone as he worked on this subject matter while employed at the Y-12 Plant. Mr. Wallace is also a named inventor with Dr. Dai, Dr. Im and Dr. Stephan on a sol-gel method of making a neutron scintillator.

Mr. Wallace has completed working on a DOE Phase I grant verifying that manufacture of the lithium- 6 phosphate nanoparticles invented at ORNL can be successfully transferred to the private sector. Neutron Sciences has licensed the technology and will be using the material in developing neutron detecting scintillation materials. The data taken during the Phase I show that the nanoparticles are a very cost effective replacement for helium-3 and fiber drawn glass neutron detectors.

Andrew C. Stephan, Sheng Dai, Steven A. Wallace and Laurence F. Miller, Modeling of composite neutron scintillators, Radiation Protection Dosimetry, 116 (2005), 165-169.

Andrew C. Stephan, Sheng Dai, Steven A. Wallace, "Neutronics aspects of position sensitive neutron scintillator detectors using wavelength-shifting readout fibers," Applied Radiation and Isotopes, 61 (2004), 1375-1382.

Steven Wallace, Andrew C. Stephan, Phillip C. Womble, Gavi Begtrup, Sheng Dai, "Spectra of fast neutrons using a lithiated glass film on silicon," Nucl. Instr. and Meth. A 505 (2003), 16-19.

Steven Wallace, Andrew C. Stephan, Laurence F. Miller, Sheng Dai, "Neutron detector based on lithiated sol-gel glass," Nucl. Instr. and Meth. A 483 (2002), 764-773.

S. A. Wallace, J. H. Hiller, S. Dai, L. F. Miller, "Neutron Detection Based Upon a Lithiated Sol-Gel Glass," Applied Radiation and Isotopes 53 (2000) 755.

Dai; Sheng; Stephan; Andrew Curtis; Brown; Suree S.; Wallace; Steven A.; and 
Rondinone; Adam J., Composite scintillators for detection of ionizing radiation, United States Patent Application 20060054863, March 16, 2006.

Wallace, Steven; Stephan, Andrew C.; Dai, Sheng; and Im, Hee-Jung; Neutron detector using lithiated glass-scintillating particle composite, United States Patent Application 20030178574, September 25, 2003.

Peter Hansen - Technical Lead for Telesensors, Inc. and Chief Technical Officer

Ph.D. 1990 Electrical Engineering University of Tennessee, Knoxville, TN

M.S. 1985 Electrical Engineering University of Tennessee, Knoxville, TN

B.S. 1978 Electrical Engineering University of Illinois, Urbana, IL

2005 - present: Chief Technology Officer at Telesensors, Inc.

Responsible for the development and acquisition of technology as well as overall supervision of current projects.

1997 - 2005: Senior Research Engineer at Flextronics, Int. (formerly ASIC, Int.). Contributed to a wide variety of digital and analog Application Specific Integrated Circuit (ASIC) design efforts.

1996 - 1997: Research Associate at the University of Tennessee, Knoxville, TN.

Created a multi-threaded client/server software application to enable viewing of very large images $(500 \mathrm{MB})$ for law enforcement applications

Patents:

Method and apparatus for the interactive display of any portion of a spherical image, US $5,990,941$.

Ultra-capacitor based dynamically regulated charge pump converter, US 6,370,046.

Dynamically controlled, intrinsically regulated charge pump, US 6,310,789.

Multiple output dynamically regulated charge pump power converter, US 6,643,151.

\section{(8) Facilities/Equipment}

No equipment should cost over five thousand dollars. The neutron source facility at the University of Tennessee Department of Nuclear Engineering will be used for the testing of the samples. Five thousand dollars is to be used to reimburse the Nuclear Engineering Department for the use of their facility. 


\section{(9) Subcontractors/Consultants}

Telesensors is the major subcontractor. Thirty five thousand dollars is to be allocated to pay for the design and fabrication of electronics. An electronics subassembly is required to interface to the Hamamatsu H7546B photomultiplier tube (PMT) module, and provide a real-time readout. There are several key features with the proposed electronics. These features include: a 64 channel analog front-end which interfaces with each channel of the H7546B PMT, pulse counters for each channel, an accumulator to aggregate data from all 64 channels, and interface circuitry to drive an LCD display. A combination of transducer fibers, PMT and electronics creates a prototype for the commercial product. Please see the figure below for a conceptual diagram.

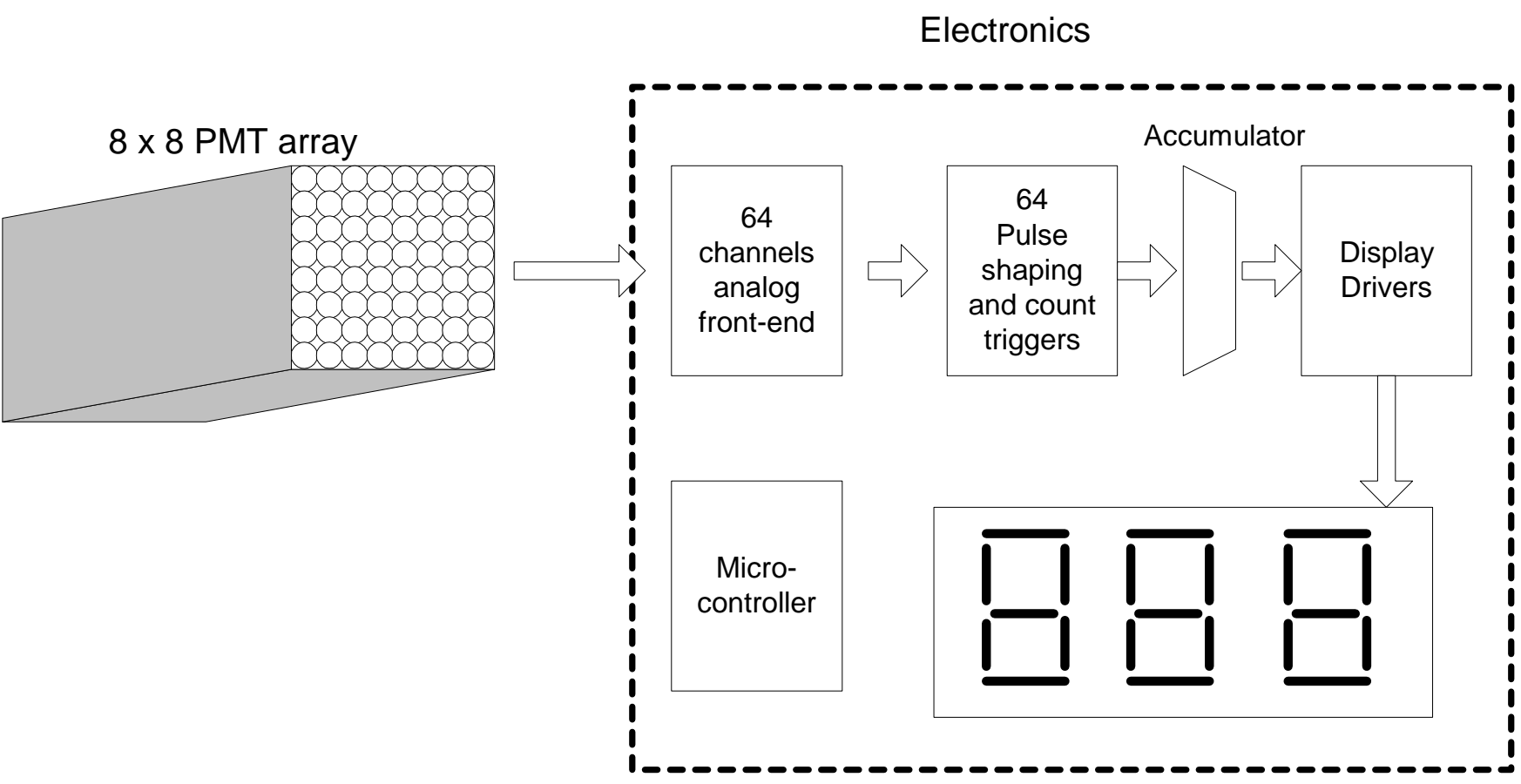

The following design criteria will guide the implementation of the prototype system. First, the 64 input channels need to be low-noise with minimal crosstalk. The electronics will be optimized for low power consumption. The ability for the electronics to operate from a battery for an extended period of time will permit more product applications. Robustness is also a key design criterion. The electronics will need to be highly integrated to reduce part counts and the number of interconnections.

Functional Operation:

Each channel of the PMT will be connected in parallel to a corresponding channel of the analog front-end. During operation, all 64 channels will operate continuously. When a pulse is received from the PMT, it will be shaped and discriminated to minimize 
the effects of noise. The accumulator will tally inputs from all 64 channels during a collection period and transfer the count to the display driver. The Liquid Crystal Display (LCD) will then provide a real-time count of the neutron flux. A short collection period will provide a fast (high variance) response. By increasing the collection period, the variance of the measured flux will be reduced, at the expense of a slower response.

The Phase I electronics will be a proof of concept only. It will have enough functionality to demonstrate the technology. However, it will not have features typically expected in a commercial product. For a Phase II design we would propose adding survey memory to hold a reasonable amount of data. This data could then be downloaded to a PC via USB cable or wireless interface. Also, in addition to the LCD read-out, the user interface could include programmable visual and audio alarms such as buzzers, flashing lights, a cell phone type vibrator, etc., to warn users of a neutron flux exceeding a predefined threshold.

About Telesensors: Telesensors is developing the next generation of intelligent wireless sensors, called Smart Sensors. Smart Sensors are low cost, wireless integrated circuits that can detect nuclear, chemical, or biological agents, as well as more conventional environmental conditions, such as temperature. Smart Sensors achieve low cost due to a high level of integration and the efficient use of the silicon die. Smart Sensors communicate within a secure wireless networked environment. These capabilities enable Smart Sensors to be deployed in the high quantities necessary to provide effective monitoring of environmental conditions. This distributed approach to environmental monitoring is far more cost-effective than conventional and expensive point-type solutions. Our mission is to take innovative Smart Sensors to market. Obviously the use of an array of neutron detectors is the application of most interest coming out of this grant. 